\title{
Anomalous ELF phenomena in the Schumann resonance band as observed at Moshiri (Japan) in possible association with an earthquake in Taiwan
}

\author{
M. Hayakawa ${ }^{1}$, A. P. Nickolaenko ${ }^{2}$, M. Sekiguchi ${ }^{1}$, K. Yamashita ${ }^{1}$, Y. Ida ${ }^{1}$, and M. Yano ${ }^{1}$ \\ ${ }^{1}$ Department of Electronic Engineering and Research Station on Seismo Electromagnetics, The University of \\ Electro-Communications, 1-5-1 Chofugaoka, Chofu Tokyo 182-8585, Japan \\ ${ }^{2}$ Usikov Institute of Radiophysics and Electronics, Ukrainian Academy of Sciences, 12, Acad. Proskura Str., \\ Kharkov 61085, Ukraine
}

Received: 10 July 2008 - Revised: 15 October 2008 - Accepted: 15 October 2008 - Published: 2 December 2008

\begin{abstract}
The ELF observation at Moshiri (geographic coordinates: $44.29^{\circ} \mathrm{N}, 142.21^{\circ} \mathrm{E}$ ) in Hokkaido, Japan, was used to find anomalous phenomena in the Schumann resonance band, possibly associated with a large earthquake (magnitude of 7.8) in Taiwan on 26 December 2006. The Schumann resonance signal (fundamental $(n=1), 8 \mathrm{~Hz} ; 2$ nd harmonic, $14 \mathrm{~Hz}, 3 \mathrm{rd}$ harmonic, $20 \mathrm{~Hz}, 4 \mathrm{th}, 26 \mathrm{~Hz}$ etc.) is known to be supported by electromagnetic radiation from the global thunderstorms, and the anomaly in this paper is characterized by an increase in intensity at frequencies from the third to fourth Schumann resonance modes mainly in the $B_{E W}$ component with a minor corresponding increase in the $B_{N S}$ component also. Spectral modification takes place only in the interval of 21:00 UT $\pm 1 \mathrm{~h}$, which corresponds to the global lightning activity concentrated in America. While distortions were absent in other lightning-active UT intervals, in particular, around 08:00 UT $\pm 1 \mathrm{~h}$ (Asian thunderstorms) and around $15 \pm 1 \mathrm{~h}$ (African lightning activity). The anomaly occurred on 23 December three days prior to the main shock. The results observed were explained in terms of ELF radio wave perturbation caused by the lower ionospheric depression around the earthquake epicenter. The difference in the path lengths between the direct radio wave from an active global thunderstorm center and the wave scattered from the non-uniformity above Taiwan causes interference at higher resonance modes, which is successful in explaining the observational data.
\end{abstract}

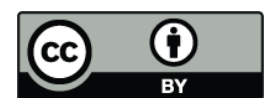

Correspondence to: M. Hayakawa (hayakawa@whistler.ee.uec.ac.jp)

\section{Introduction}

Schumann resonance (SR) is the global electromagnetic phenomenon observed in the Earth - ionosphere cavity in the ELF frequency band (Nickolaenko and Hayakawa, 2002). It is excited by electromagnetic radiation from global lightning discharges and is used for monitoring the thunderstorms worldwide (see e.g. Nickolaenko and Hayakawa, 2007; Sekiguchi et al., 2008).

The peak frequencies of power spectra of SR are $8 \mathrm{~Hz}$ (the fundamental mode $n=1), 14 \mathrm{~Hz}(n=2), 20 \mathrm{~Hz}(n=3), 26 \mathrm{~Hz}$ $(n=4)$, etc. Amplitudes of resonant oscillations vary by a factor of 2-3 during the day in response to the motion of global lightning activity, while the peak frequencies change only by a few percents (Nickolaenko and Hayakawa, 2002). Thus, the latter nature of SR enables us to use SR as a probe of the lower ionosphere just like VLF/LF radio signals of fixed frequency (Hayakawa, 2007; Molchanov and Hayakawa, 2008).

An anomalous effect in SR band has been reported for the first time by Hayakawa et al. (2005), who have demonstrated an abrupt enhancement around the fourth mode combined with a significant frequency shift (by more than $1 \mathrm{~Hz}$ ) from its conventional value of $26 \mathrm{~Hz}$. The effect was detected at Nakatsugawa near Nagoya in Japan, being associated with a large Chi-chi earthquake (EQ) in Taiwan. Ohta et al. (2006) have further performed a statistical study of anomalous spectra of SR collected at Nakatsugawa for many Taiwan EQs, who have confirmed a clear relationship between SR modifications and the seismic activity in Taiwan. An explanation has been suggested by Hayakawa et al. (2005) that spectral modifications arise from the wave interference between the direct radio wave arriving at an observer from the global thunderstorms and the wave scattered

Published by Copernicus Publications on behalf of the European Geosciences Union. 
(a) Global map with the Moshiri observatory in the center

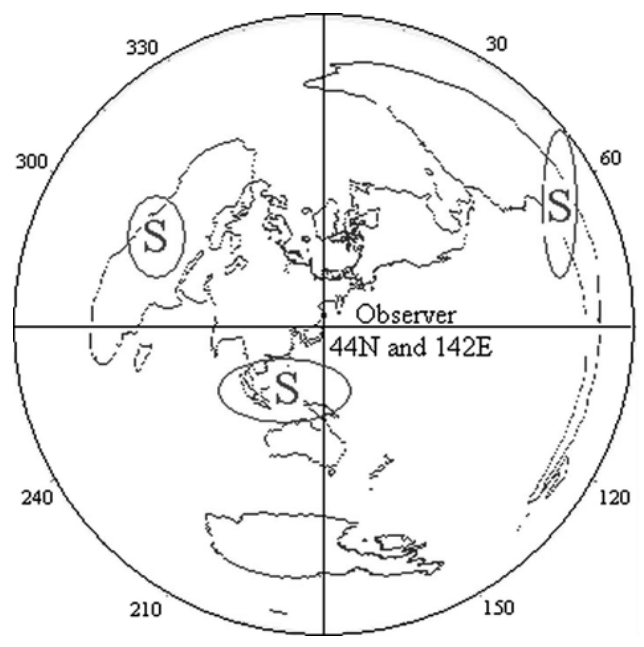

(b) Vicinity of observatory and Taiwan

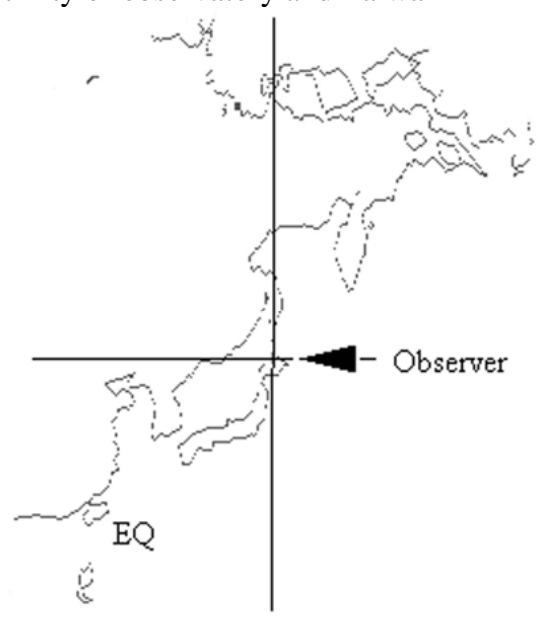

Fig. 1. Global map centered at the Moshiri observatory. (a) Positions of three global thunderstorm centers in America, Africa, and Asia are shown as ellipses with letter S inside. (b) The observatory and EQ epicenter in Taiwan.

by the seismo-ionospheric perturbation. The plasma nonuniformity occurs in the lower ionosphere positioned just above the epicenter of a future EQ in Taiwan.

The present paper is a further development of the above concept of unusual SR records associated with EQs. We analyze the time period around a large EQ in Taiwan occurred on 26 December 2006. Our observation site is placed in Japan again, but not at Nakatsugawa (around the center of Honshu, the main island of Japan) as in previous studies (Hayakawa et al., 2005; Ohta et al., 2006). We use the ELF data from an observatory at Moshiri in Hokkaido, the northern island of Japan (geographic coordinates: $44.29^{\circ} \mathrm{N}, 142.21^{\circ} \mathrm{E}$ ).

\section{EQ treated in this paper}

A large EQ took place in Taiwan on 12:26 UT (21:26 LT) on 26 December 2006. The geographic coordinates of its epicenter were $22.00^{\circ} \mathrm{N}$ and $120.48^{\circ} \mathrm{E}$, and the magnitude of the main shock was 7.3 and the depth was $10 \mathrm{~km}$. There was also a strong aftershock at 12:40 UT on the same day, with the magnitude $\mathrm{M}=5.6$ and the depth of $10 \mathrm{~km}$.

\section{ELF observation in Japan}

At the time of this EQ the ELF observatory at Nakatsugawa was out of service, where the SR anomaly was first observed in association with the EQs in Taiwan (Hayakawa et al., 2005; Ohta et al., 2006), and the ELF equipment there was being upgraded at that time. However, the ELF station was fortunately working good at another station at Moshiri, Hokkaido. We use below the ELF data recorded there: the waveforms of two orthogonal horizontal magnetic field components $B_{N S}, B_{E W}$ (subscripts NS and EW mean north-south and east-west, respectively) and of the vertical electric field $\mathrm{E}_{Z}$. Monitoring with the sampling frequency of $4 \mathrm{k} \mathrm{Hz}$ has been continued since July 2004. Details of the ELF observations at Moshiri are found in Ando et al. (2005) and Matsudo et al. (2007).

\section{Anomalous ELF effects observed at Moshiri}

Figure 1 depicts the global map with the origin at the observatory of Moshiri. It shows three global thunderstorm centers in America, Africa, and Asia marked as ellipses with letter S (source) inside. Lightning strokes of each center contribute to the SR signal at different times of day (Nickolaenko and Hayakawa, 2002) owing to the delay in maxima of their activity. Asian thunderstorms play the dominant role around $\mathrm{UT}=(8 \pm 1) \mathrm{h}$, the global activity shifts to Africa at $\mathrm{UT}=(15 \pm 1) \mathrm{h}$, and to America around $(21 \pm 1) \mathrm{h}$. The natural ELF radio signals tend to arrive at an observer from different directions during the day (Nickolaenko and Hayakawa, 2002). The lower panel (Fig. 1b) indicates the geometry of our observatory and the EQ epicenter in Taiwan.

Figure 2 shows dynamic spectra of horizontal magnetic field components $B_{N S}$ (upper panel) and $B_{E W}$ (lower panel) for the particular interval UT $=21 \pm 1 \mathrm{~h}$ (when American source dominates) for the one-month period around the EQ date (the day of EQ is marked by the black box). Individual spectra corresponding to the relevant $2 \mathrm{~h}$ time intervals are presented in Fig. 2 day after day. One may note in Fig. 2 that the $B_{E W}$ intensity (lower panel) is considerably enhanced in the frequency range above $10 \mathrm{~Hz}$ on 23 December being simultaneously depressed below $10 \mathrm{~Hz}$. These deviations will be discussed later in a quantitative way.

In order to make this anomaly seen in Fig. 2 much more convincing, we have performed the following signal analysis. 

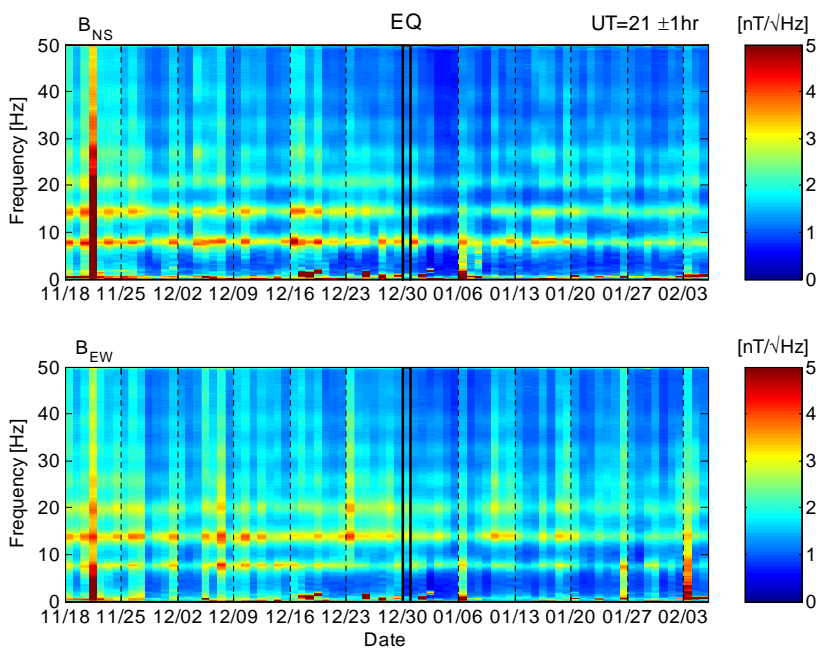

Fig. 2. Extremely low frequency dynamic spectra for the time interval $\mathrm{UT}=21 \pm 1 \mathrm{~h}$ when the American thunderstorms dominate in the Earth - ionosphere cavity. The upper panel presents the power spectra of the $B_{N S}$ field component, and the bottom presents the $B_{E W}$ spectra. The period covers from 18 November 2006 to the beginning of February 2007. The EQ date is given by the black box.

We have examined temporal evolutions of frequency spectra of two horizontal magnetic field components $B_{N S}$ and $B_{E W}$ during one year, \pm 6 months around the EQ on 26 December 2006. For each $2 \mathrm{~h}$ time interval for three lightning sources (America, Africa and Asia) of a given day, we estimate the running average power spectrum $(<\mathrm{B}(\mathrm{f})>)$ in the frequency band of $2.5-40 \mathrm{~Hz}$ for that day. That is, the spectrum averaged over \pm 15 days (total 31 days) just around the relevant day, is regarded as a reference spectrum for that day. Then, we make a difference between the frequency spectrum for this particular day $(\mathrm{B}(\mathrm{f}))$ and the corresponding running average for this day $(<\mathrm{B}(\mathrm{f})>)$, and we obtain the difference (residue) $\Delta \mathrm{B}(\mathrm{f})=\mathrm{B}(\mathrm{f})-<\mathrm{B}(\mathrm{f})>$ for this day.

We have examined all of the three lightning sources (America, Africa, Asia), and we have found that the relevant difference (residue) spectra $\Delta B_{E W}(f)$ and $\Delta B_{N S}(f)$ show no remarkable anomaly for other time intervals of African source (UT $=15 \pm 1 \mathrm{~h})$ and of Asian source $(\mathrm{UT}=8 \pm 1 \mathrm{~h})$. Figure 3 illustrates the dynamic spectra of $\Delta B_{N S}(f)$ (top) and $\triangle B_{E W}(f)$ (bottom) for the UT interval of $21 \pm 1 \mathrm{~h}$. A significant anomaly is clearly seen in Fig. 3 only when the global thunderstorm activity moves to America (UT=21 $\pm 1 \mathrm{~h}$ ).

Figure 4 compares particular spectra of SR during normal and abnormal days for the American source (UT $=21 \pm 1 \mathrm{~h}$ ). Here, the blue lines in frames (a) and (b) depict the power spectra observed on a quiet day of 21 December. Frames (c) and (d) refer to the day with anomaly on 23 December. The red lines in frames (a)-(d) depict the running mean spectra. As already described before, these were averaged over \pm 15 days around the dates of 21 and 23 December. Plots (e)-(h)
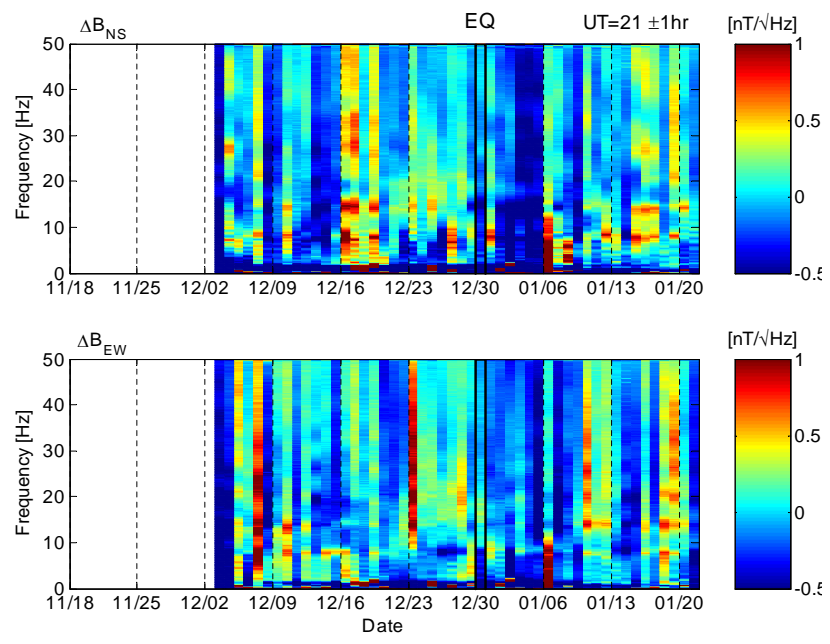

Fig. 3. Dynamic spectra of the field deviations $\Delta B_{N S}(f)$ and $\triangle B_{E W}(f)$ relevant to the time interval of $\mathrm{UT}=21 \pm 1 \mathrm{~h}$. The upper panel shows deviations $\Delta B_{N S}(f)$ and the lower is the $\Delta B_{E W}(f)$ spectra. An anomaly on 23 December is seen as vertical red-brown bars in the frequency above $10 \mathrm{~Hz}$.

show the spectra $\Delta B_{E W}(f)$ and $\Delta B_{N S}(f)$ in order to find out any EQ signature. Thus, (a) and (b) frames of Fig. 4 show the power spectra $B_{N S}$ and $B_{E W}$ in quiet conditions, while the (c) and (d) spectra show the anomalous signal. That is, one may observe that deviations appear at frequencies above $10 \mathrm{~Hz}$ with an apparent enhancement at the $2 \mathrm{nd}$ and $3 \mathrm{rd}$ of SR modes on the anomalous day of 23 December. Modification in the $B_{N S}$ field component is also present, but it is less pronounced than that in the $B_{E W}$ field. Plots (e)-(h) of Fig. 4 show deviations of power spectra from the monthly running average. The horizontal straight lines here depict the $\sigma$ and $2 \sigma$ levels where $\sigma$ is the standard deviation of the power spectra in the $2.5-40 \mathrm{~Hz}$ frequency range for the whole month period. These lines demonstrate the statistical significance of spectral anomaly. Modifications in the plots (g) and (h) substantially exceed the $2 \sigma$ level, and we must conclude that this anomaly is far beyond the usual fluctuations of SR intensity. On the other hand, plots (e) and (f) illustrate the spectral deviations on 21 December, which never go out from the $2 \sigma$ threshold and must be attributed to customary fluctuations caused by statistical nature of the global thunderstorm activity.

The spectral anomaly was apparent only in the UT interval of $21 \pm 1$ h, i.e. when American thunderstorms dominate in the Earth - ionosphere cavity. In order to reconfirm this fact, Fig. 5 presents the corresponding difference spectra similarly obtained for other two intervals of UT $=8 \pm 1 \mathrm{~h}$ (Asian thunderstorms) and UT $=15 \pm 1 \mathrm{~h}$ (African activity) on the same "active" day of 23 December. One may observe that no anomaly is present in these UT sectors. 


\section{Interpretation of anomalous ELF effects}

Similarly to Hayakawa et al. (2005), we interpret the abnormal ELF radio signal as an interference of direct and scattered radio waves. Figure 6 shows schematically the relative location of the disturbance over Taiwan and the observer at Moshiri together with parameters of propagation paths. Three global thunderstorm centers are shown: Asia $\left(0^{\circ} \mathrm{N}\right.$ and $\left.120^{\circ} \mathrm{E}\right)$, Africa $\left(5^{\circ} \mathrm{N}\right.$ and $\left.10^{\circ} \mathrm{E}\right)$, and America $\left(0^{\circ} \mathrm{N}\right.$ and $50^{\circ} \mathrm{W}$ ). It follows from the geometry of Fig. 6 that only American thunderstorms are able to provide an anomaly in the SR band. Owing to the path orientation, the major modifications will occur in the $B_{E W}$ field with the corresponding smaller effect on the $B_{N S}$ component, exactly as it was observed in the experiment.

Geometrical considerations indicate that the time of noticeable changes in resonance spectra must coincide with the period of American thunderstorms, i.e. UT=around $21 \mathrm{~h}$, which also coincides with the measurements. A difference in the direct and scattered path lengths is the most important quantity for the wave interference: it controls the frequencies where spectral modifications might take place. The relevant path difference was as follows: $0.23 \mathrm{Mm}$ for Asian thunderstorms, $2.4 \mathrm{Mm}$ for African activity, and 5.7 Mm for American lightning strokes, as seen in Fig. 6. The longest path difference corresponds to American sources, so that modifications might occur in the SR band only around UT $\cong 21 \mathrm{~h}$.

Then we computed direct, scattered and the total vertical electric field components in the disturbed Earth - ionosphere cavity when the global thunderstorms concentrate in one of three centers shown in Fig. 6. When introducing an ionospheric perturbation associated with the seismic activity, we located the depression above the future EQ focus, assumed that the lower ionosphere was reduced in height as a whole by $20 \mathrm{~km}$, accepted a Gaussian radial dependence of perturbation, and found the relevant localized modification of the ELF field. The characteristic size of disturbance was chosen to be $1000 \mathrm{~km}$. Further details of the modeling the nonuniformity and ELF radio propagation might be found either in Appendix, in Hayakawa et al. (2005) or in Nickolaenko et al. (2006).

Figure 7 depicts the computed frequency spectra relevant to separate thunderstorm centers of America (upper panel), Africa (middle panel), and Asia (bottom penel). The thin lines here show the spectra of direct wave, i.e. SR expected in the uniform cavity with the given source position. The thick lines refer to the ionospheric non-uniformity over Taiwan. Owing to small differences in the path lengths for African and Asian thunderstorms, substantial spectral changes occur well above the SR band in the frequency range above $30 \mathrm{~Hz}$. A significant path difference for the American source provides modifications within the SR band, as is demonstrated in Fig. 7. There is an increase around $10 \mathrm{~Hz}$, a decrease from 16 to $20 \mathrm{~Hz}$, and an enhancement from 20 to $30 \mathrm{~Hz}$. Of course, the agreement between our simple theoretical estimation and the observation is not complete, but we can conclude that the frequency dependence in the top panel of Fig. 7 is consistent in general with the observation presented in Figs. $4 \mathrm{c}$ and $4 \mathrm{~d}$. The discrepancy might arise from the following reasons:

1. computations refer to the vertical electric field component, while horizontal magnetic fields are measured in the experiment,

2. point sources were used in the model, and the exact location and the size of zone occupied by global thunderstorms is unknown for the moment of measurements,

3. the characteristics of ionospheric modification by preseismic activity might deviate from that assumed in our model.

We listed obvious factors that might alter the spectral modifications, but the major features of pre-seismic effects detected experimentally are also present in the model. In particular, significant changes of frequency spectra occur at higher resonance modes in the SR frequency range from 20 to $30 \mathrm{~Hz}$.

\section{Summary and discussion}

In our previous study by Hayakawa et al. (2005), the anomalous SR effects were recorded at the Nakatsugawa observatory in possible association with EQs in Taiwan. The anomaly was characterized by a narrow-banded nature around the fourth resonance frequency in the form of a high sharp peak shifted from the regular $26 \mathrm{~Hz}$ frequency. While, significantly different characteristics are presented in this paper, as based on the ELF observation at Moshiri, in association with an EQ again in Taiwan; that is, a bias at frequencies above $10 \mathrm{~Hz}$ and in the frequency range of $20-30 \mathrm{~Hz}$. The model ionospheric modification in this paper is exactly the same as in Hayakawa et al. (2005), but the fundamental nature of the difference between this paper and previous paper by Hayakawa et al. (2005) is due to a much larger optical path difference in the present paper.

Stimulated by the work by Hayakawa et al. (2005), Schekotov et al. (2007) have tried to find any effects in the ELF data collected at Kamchatka. No similar signals as in Hayakawa et al. (2005) were detected there in the SR band, but other peculiarities were found instead. That is, ELF emissions appeared below the SR fundamental mode $(8 \mathrm{~Hz})$ associated with local seismic activity. These seismogenic ULF/ELF emissions occupied the frequency band of 4-6 Hz and were detected as an enhanced $\mathrm{Phh} / \mathrm{Pdd}$ power spectra ratio (The horizontal magnetic components were measured along (h) and across (d) the local magnetic meridian). An increase in the ratio of Phh/Pdd was accompanied by a reduction in the standard deviation of the orientation angle of the polarization ellipse. The area of these seismogenic ELF modifications was estimated to be detected within a 
$\mathrm{UT}=21 \pm 1 \mathrm{~h}$
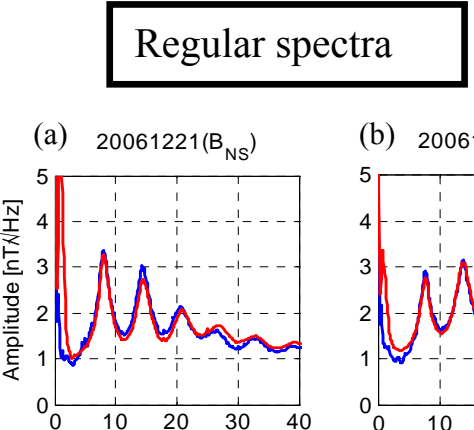

(e)

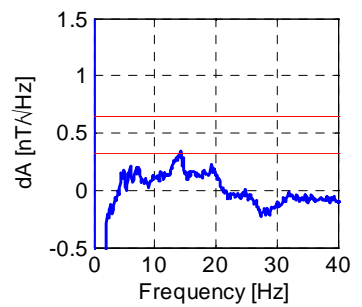

(b) 20061221( $\left.\mathrm{B}_{\mathrm{EW}}\right)$

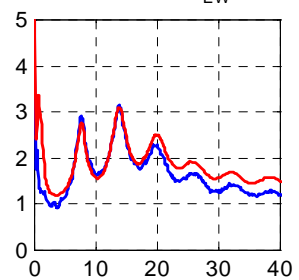

(f)

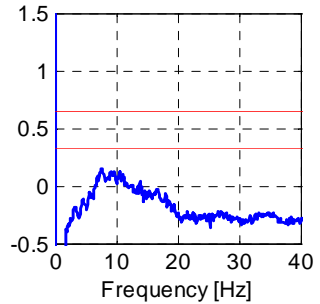

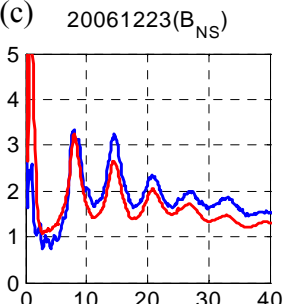

(g)

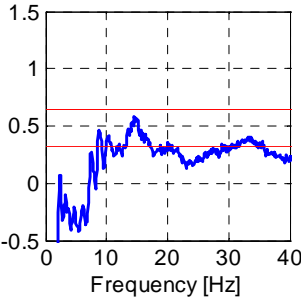

Anomalous spectra

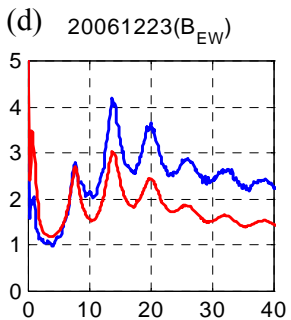

(h)

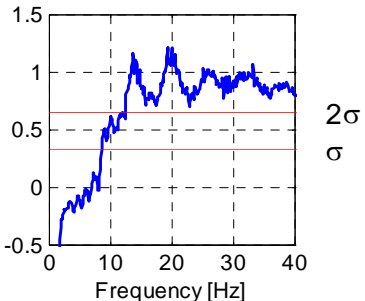

Fig. 4. Particular power spectra of ELF radio signal recorded in the time interval UT $=21 \pm 1 \mathrm{hr}$. Panels (a) and (b) show the spectrum of $B_{N S}$ and $B_{E W}$ field components on a regular (undisturbed) day of 21 December. The red line is the monthly averaged power spectrum, while the blue line is the spectrum of the particular day. Panels (c) and (d) present spectra $B_{N S}$ and $B_{E W}$ on the disturbed day of 23 December. The red line is the monthly averaged power spectrum, and the blue line is the spectrum of the particular day. Panels (e)-(h) depict spectral differences $\Delta B_{N S}$ and $\Delta B_{E W}$ on the quiet (21 December) and disturbed (23 December) days. The standard deviation $\sigma$ and the $2 \sigma$ level are indicated by horizontal straight lines.

few hundred kilometers from the future EQ epicenter. Ohta et al. (2001) had already observed at Nakatsugawa (Japan) similar seismogenic ULF/ELF emissions possibly associated with the great Chi-chi EQ in Taiwan. The emissions covered a wide frequency band, up to a few tens of $\mathrm{Hz}$, although the frequency spectrum was not examined thoroughly.

An anomaly described in the present study takes place above the SR fundamental frequency, and we do not address here the modifications present at frequencies below $1 \mathrm{~Hz}$ as seen in Fig. 4. "High frequency" modifications can hardly be explained in terms of generation of the wide-banded electromagnetic noises directly by a seismic source just like it was suggested for the ULF band (Fraser-Smith et al., 1990; Kopytenko et al., 1993; Hayakawa et al., 1996). The reason is that the skin-depth at the higher SR modes excludes any possibility of subsurface signal propagation through the ground soil to the atmosphere. To make information complete, we mention that the ELF noise corresponding to the anomaly observed on 7 December 2006 (see Fig. 3) was composed of many frequent pulses. However, the seismic origin of these impulses seems dubious.

It looks reasonable to attribute the anomalies detected in this paper to changes in the lower ionosphere above the future EQ focus and to the interference of direct and scattered ELF radio signals. The major field source was the lightning activity in America as is evidenced by the major effect on $B_{E W}$ and the signals were modified by reflections from the ionospheric non-uniformity caused by the pre-seismic activity in Taiwan. Computed modifications of SR spectra agree with the observations. No distinct effect was observed when thunderstorms concentrate in Asian and African sectors, in consistence with the prediction of the model. Besides, the model indicates absence of the considerable effect at frequencies below $10 \mathrm{~Hz}$ since the path length difference between the direct and scattered signals is small enough for all the sources. Therefore we conclude that the experimental evidence presented here might be reasonably explained by model computations, and both of them indicate a realistic modification of the lower ionosphere (a depression) above the center of future EQ.

To conclude the paper, we list the similarity and distinctions of our results in comparison with published data by Hayakawa et al. (2005) and Ohta et al. (2006).

\subsection{Similarity}

Spectral anomalies in the SR band are observed in Japan in association with seismic activity in Taiwan.

Modifications tend to occur at higher SR modes. 


\subsubsection{Precursor spectra}

(a) $\mathrm{UT}=8 \mathrm{hr}\left(\mathrm{B}_{\mathrm{NS}}\right)$

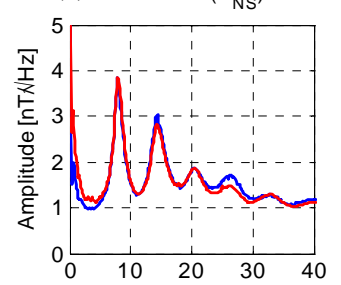

(e)

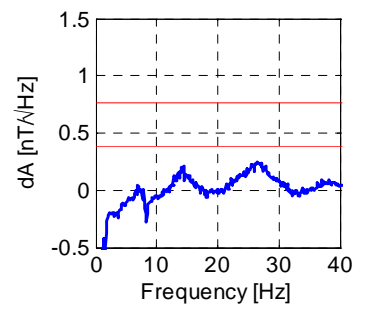

(b) $8 \mathrm{hr}\left(\mathrm{B}_{\mathrm{EW}}\right)$

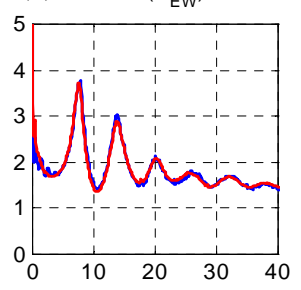

(f)

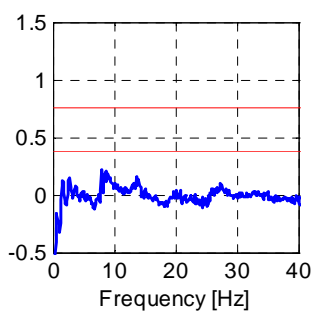

(c) $\mathrm{UT}=15 \mathrm{hr}\left(\mathrm{B}_{\mathrm{NS}}\right)$

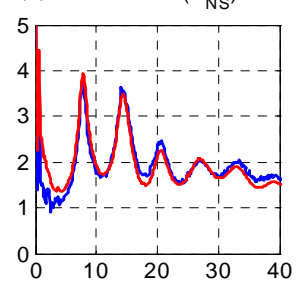

(g)

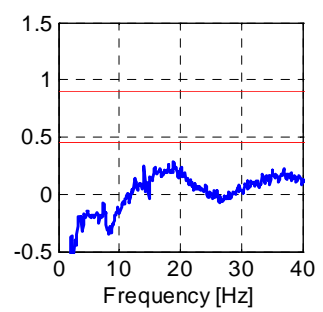

(d) $15 \mathrm{hr}\left(\mathrm{B}_{\mathrm{EW}}\right)$

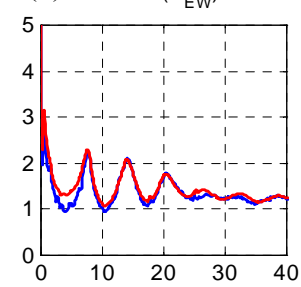

(h)

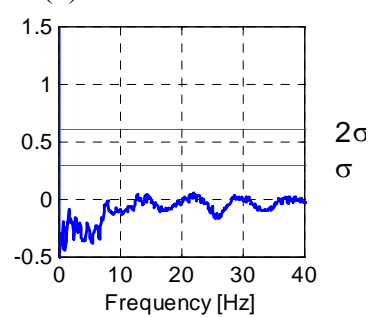

Fig. 5. Power spectra on the anomalous day of 23 December 2006 , recorded in the time intervals UT=8 $\pm 1 \mathrm{~h}$ (left plots) and UT=15 $\pm 1 \mathrm{~h}$ (right plots). Panels (a)-(d) show correspondingly the spectra $B_{N S}$ and $B_{E W}$ for UT=8 \pm 1 (Asian source) and $15 \pm 1 \mathrm{~h}$ (African source). The red line is the monthly averaged power spectrum, while the blue line is the spectrum of particular day. Panels (e)-(h) show the spectral differences $\Delta B_{N S}$ and $\Delta B_{E W}$ for $\mathrm{UT}=8 \pm 1 \mathrm{~h}$ and $15 \pm 1 \mathrm{~h}$, correspondingly. Horizontal lines indicate levels of $\sigma$ and the $2 \sigma$.

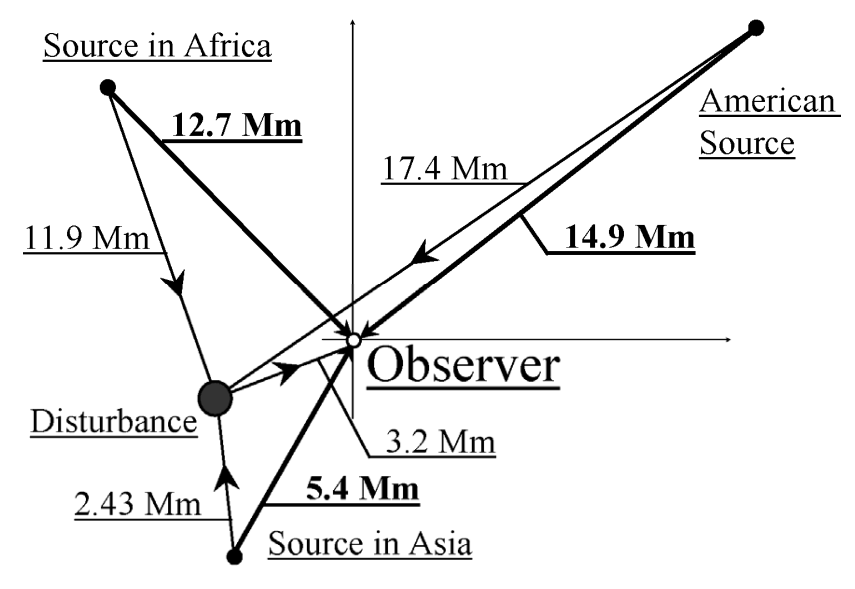

Fig. 6. Relative position of three global thunderstorm centers, the ELF observatory at Moshiri, and the ionospheric disturbance over Taiwan. The optical lengths are shown of the direct and scattered propagation paths.

The "arrival angle" of modifying factor agrees with the direction toward the EQ epicenter.

\subsection{Distinctions}

Effects observed at Moshiri were short-term modifications, which were detected only on one day and in a narrow UT interval of $21 \pm 1 \mathrm{~h}$.
Spectral modifications at Moshiri have a clear precursory character. They occurred three days prior to the main shock and were absent afterwards (In this context, they look similar to the ULF precursors of Spitak, Loma-Prieta and Guam EQs).

Anomalous signals at Moshiri occupy a wide frequency band, while anomalies reported before had a narrow-band nature.

\section{Appendix A}

We used the following model in our computations. We treat the vertical electric field for simplicity. The primary radio wave $\left(E_{1}\right)$ arrives at an observer together with the wave scattered by a localized ionospheric disturbance wave $\left(E_{2}\right)$, so that the total field $(E)$ is a sum of these two waves.

$E=E_{1}+E_{2}$

The direct wave is found from Nickolaenko and Hayakawa (2002),

$E_{1}(\omega)=\frac{M(\omega)}{4 h a^{2} \varepsilon} \frac{i \nu(\nu+1)}{\omega} \frac{P_{\nu}\left[\cos \left(\pi-\theta_{H}\right)\right]}{\sin \pi \nu}$,

where $\omega=2 \pi f$ is the circular frequency, $M(\omega)$ is the source current moment, $v(\omega)$ is the propagation constant, $h$ is the 
$\left|\mathrm{E}_{\mathrm{r}}(f)\right|$ a.u.
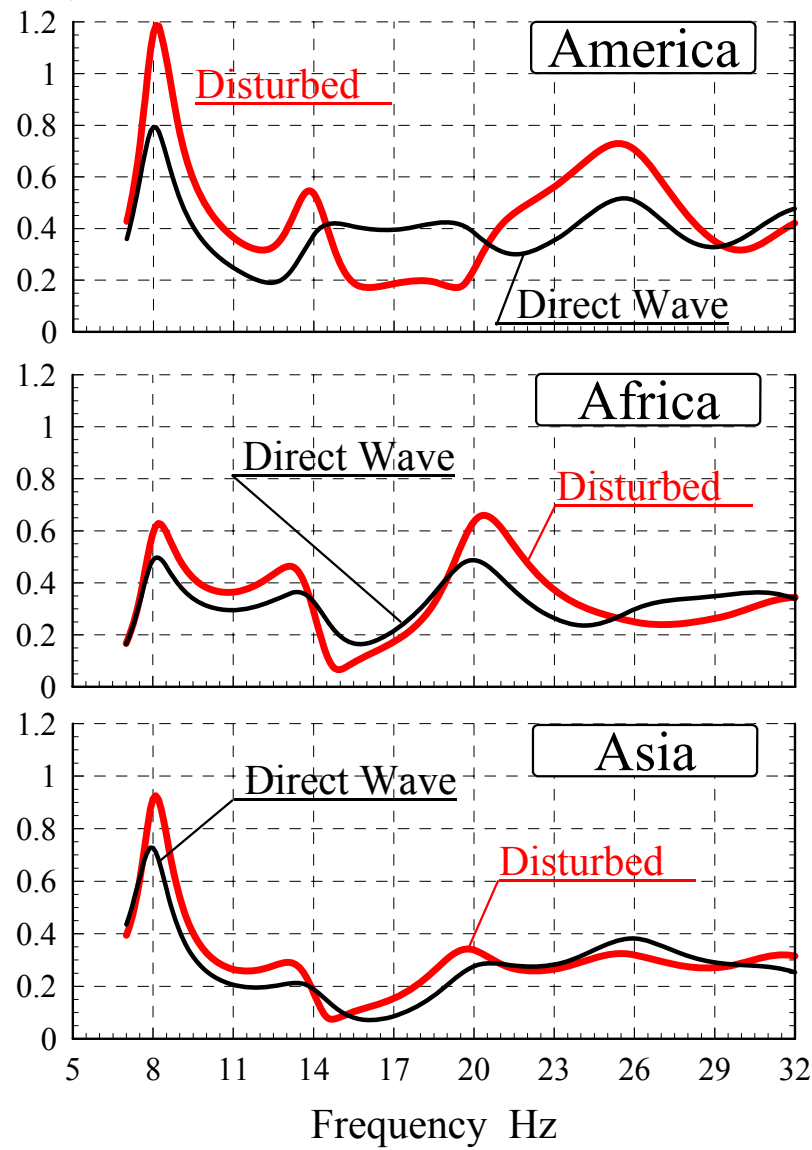

Fig. 7. Regular or undisturbed (black line) and disturbed (red line) amplitude spectra of $\left|E_{z}(f)\right|$ field component computed for thunderstorms concentrated at America (upper plot), Africa (middle plot), or Asia (lower plot).

ionospheric height, $a$ is the Earth's radius, $\varepsilon$ is the dielectric constant of free space, $P_{v}(\cos \theta)$ is the Legendre function, and $\theta_{H}$ is the angular source - observer distance. The function of $v(f)$ is pertinent to the regular ionospheric profile, which is defined by the standard equations (Nickolaenko and Hayakawa, 2002): $v=-\frac{1}{2}+\sqrt{\frac{1}{4}+\left(k a S_{\nu}\right)^{2}}(k$ : free space propagation constant), $S_{v}^{2}=\frac{H_{M}}{H_{E}}\left(H_{M}\right.$ and $H_{E}$ are the ELF wave reflection height for magnetic and electric fields, respectively), and $C_{v}^{2}=1-S_{v}^{2}$. The subscript D of such as $v_{D}$ indicates the disturbed ionospheric profile.

According to Williams et al. (2006), the electric (lower) characteristic height $\left(H_{E}\right)$ in the knee model of ionospheric conductivity profile is found from the following equation.

$$
\begin{aligned}
H_{E}(f)= & H_{\mathrm{KNEE}}+\varsigma_{a} \ln \left(\frac{f}{F_{\mathrm{KNEE}}}\right)+0.5\left(\varsigma_{a}-\varsigma_{b}\right) \\
& \ln \left[1+\left(\frac{F_{\mathrm{KNEE}}}{f}\right)^{2}\right]+
\end{aligned}
$$

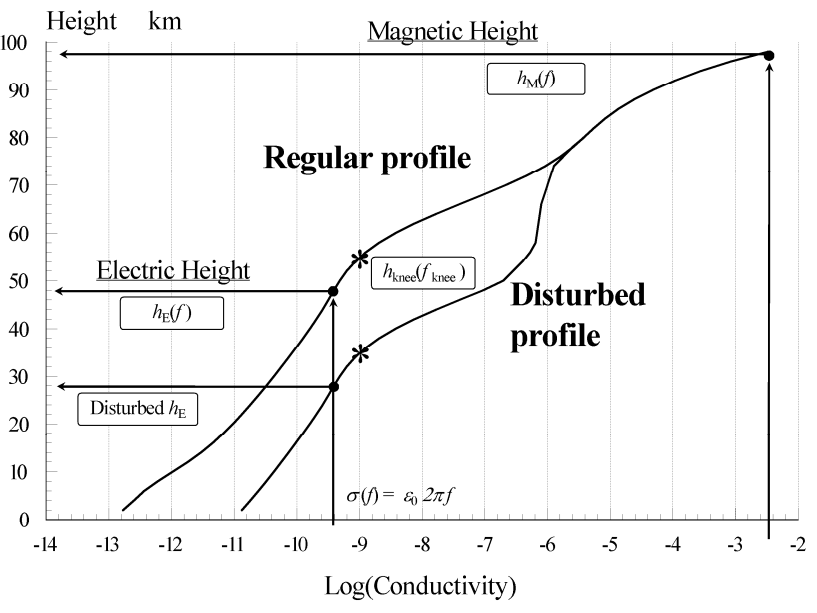

Fig. A1. Depression of the ionosphere profile right above the focus of Taiwan EQ.

$$
i\left[\frac{\pi}{2} \varsigma_{a}-\left(\varsigma_{a}-\varsigma_{b}\right) \tan ^{-1}\left(\frac{F_{\mathrm{KNEE}}}{f}\right)\right]
$$

The knee altitude $H_{\mathrm{KNEE}}=55 \mathrm{~km}$ and the frequency $F_{\mathrm{KNEE}}=10 \mathrm{~Hz}$, as seen in Fig. A1. This means that at the altitude of $55 \mathrm{~km}$ the displacement current of $10 \mathrm{~Hz}$ is equal to the conduction current. The profile has different height scales $\zeta_{b}=8.3 \mathrm{~km}$ and $\zeta_{a}=2.9 \mathrm{~km}$ below and above the knee altitude, respectively.

The magnetic (upper) characteristic height $\left(H_{M}\right)$ and its height scale are given by,

$$
\begin{aligned}
& H_{M}=H_{M P}-\varsigma_{M} \ln \left(\frac{f}{f_{M P}}\right)-i \frac{\pi}{2} \varsigma_{M} \\
& \varsigma_{M}=\varsigma_{M P}+B_{M}\left(\frac{1}{f}-\frac{1}{f_{M P}}\right),
\end{aligned}
$$

where the upper height parameters are left unchanged by the disturbance: $H_{M P}=96.5 \mathrm{~km}, \zeta_{M P}=4 \mathrm{~km}, f_{M P}=8 \mathrm{~Hz}$, $B_{M}=20 \mathrm{~km}$.

In the ionospheric depression due to the pre-seismic effect shown in Fig. A1, only a single parameter varies, i.e. the knee height, and it becomes equal to $H_{K D}=35 \mathrm{~km}$. In other words, only the lower part of profile goes down by $20 \mathrm{~km}$ as the whole, as seen in Fig. A1.

The disturbed electric height is similar to Eq. (3) with a new knee height $H_{K D}$ only:

$$
\begin{aligned}
H_{E D}= & H_{K D}+\varsigma_{a} \ln \left(\frac{f}{F_{\mathrm{KNEE}}}\right)+0.5\left(\varsigma_{a}-\varsigma_{b}\right) \\
& \ln \left[1+\left(\frac{F_{\mathrm{KNEE}}}{f}\right)^{2}\right] \\
& +i\left[\frac{\pi}{2} \varsigma_{a}-\left(\varsigma_{a}-\varsigma_{b}\right) \tan ^{-1}\left(\frac{F_{\mathrm{KNEE}}}{f}\right)\right]
\end{aligned}
$$


The disturbed complex sine and cosine functions are equal to $S_{v D}^{2}=\frac{H_{M}}{H_{E D}}$ and $C_{v D}^{2}=1-S_{v D}^{2}$, so that a change of the complex cosine propagation parameter is $\Delta C_{v}^{2}=C_{v}^{2}-C_{v D}^{2}$.

The relevant field modification is expressed through an effective change $d Z=\frac{\pi}{2} d C_{v}^{2} \cdot d^{2}$ of the surface impedance (the "disturbed" boundary condition is held at the undisturbed altitude) where $d$ is the size of the disturbance with the Gaussian spatial dependence $\delta C_{\nu}^{2}=\Delta C_{\nu}^{2} \exp \left(\frac{\cos \beta-1}{d^{2}}\right), \beta$ is the angular distance from the center of ionospheric modification, and $\alpha$ is the internal azimuth counted from the direction to the field source. We accept $d=\pi / 40$ radians, hence $d=1000 \mathrm{~km}$. For a small (in wavelengths) localized nonuniformity, the normalized disturbance is Nickolaenko and Hayakawa (2002):

$B_{Z}=\frac{d Z \cdot Q_{1}}{\sin (\pi v) P_{v}\left[\cos \left(\pi-\theta_{H}\right)\right]}$

where

$$
\begin{aligned}
Q_{1}= & v(\nu+1) P_{\nu}[\cos (\pi-\theta)] P_{v}[\cos (\pi-\gamma)] \\
& -M \cdot P_{\nu}^{1}[\cos (\pi-\theta)] P_{\nu}^{1}[\cos (\pi-\gamma)],
\end{aligned}
$$

$P_{v}^{1}(\cos \theta)$ is the associated Legendre function, $\theta$ is the angular distance from the source to the disturbance and $\gamma$ is the angular distance from the disturbance to the observer. The geometrical differentiation parameter is $M=\frac{\partial \gamma}{\partial \theta}=\frac{\sin \theta \cos \gamma \cdot \cos \alpha-\sin \gamma \cos \theta}{\sin \gamma}$. The disturbed vertical electric field is given by

$$
E^{\text {DIST }}=\left|1+B_{Z}\right|
$$

We placed this kind of disturbance over Taiwan at the geographic coordinates $\left(21.83^{\circ} \mathrm{N}\right.$ and $\left.120.54^{\circ} \mathrm{E}\right)$ and supposed that a single of three global thunderstorm centers drives the SR signal at the moment. After computing the normalized disturbance $B_{Z}(f)$, we find the unperturbed (Eq. A2) and perturbed Eq. (A8) fields and relevant power spectra shown in Fig. 7.

Acknowledgements. The authors express their sincere gratitude to NiCT (R\&D promotion scheme funding of international joint research) for its financial support.

Edited by: P. F. Biagi

Reviewed by: two anonymous referees

\section{References}

Ando, Y., Maltsev, P., Sukhynyuk, A., Goto, T., Yamauchi, T., Hobara, Y., Sekiguchi, M., Ikegami, Y., Sera, M., Korepanov, V., and Hayakawa, M.: New ELF observation system at Moshiri, Japan and assessment of acquired data, J. Atmos. Electr., 25, 29$39,2005$.
Fraser-Smith, A. C., Bernardi, A., McGill, P. R., Ladd, M. E., Helliwell, R. A., and Villard Jr., O. G.: Low frequency magnetic field measurements near the epicenter of the Loma-Prieta earthquake, Geophys. Res. Lett., 17, 1465-1468, 1990.

Hayakawa, M., Ohta, K., Nickolaenko, A. P., and Ando, Y.: Anomalous effect in Schumann resonance phenomena observed in Japan, possibly associated with the Chi-chi earthquake in Taiwan, Ann. Geophys., 23, 1335-1346, 2005, http://www.ann-geophys.net/23/1335/2005/.

Hayakawa, M.: VLF/LF radio sounding of ionospheric perturbations associated with earthquakes, Sensors, 7, 1141-1158, 2007.

Hayakawa, M., Kawate, R., Molchanov, O. A., and Yumoto, K.: Results of ultra-low-frequency magnetic field measurements during the Guam earthquake of 8 August 1993, Geophys. Res. Lett., 23, 241-244, 1996.

Kopytenko, Yu. A., Matiashvili, T. G., Voronov, P. M., Kopytenko, E. A., and Molchanov, O. A.: Detection of ultra-low frequency emissions connected with the Spitak earthquake and its aftershock activity based on geomagnetic pulsation data at Dusheti and Vardzija observatories, Phys. Earth Planet. Inter., 11, 85-95, 1993.

Matsudo, Y., Suzuki, T., Hayakawa, M., Yamashita, K., Ando, Y., Michimoto, K., and Korepanov, V.: Characteristics of Japanese winter sprites and their parent lightning as estimated by VHF lightning and ELF transients, J. Atmos. Solar-Terr. Phys., 69, 1431-1446, 2007.

Molchanov, O. A. and Hayakawa, M.: Seismo Electromagnetics and Related Phenomena: History and latest results, TERRAPUB, Tokyo, 189 p., 2008.

Nickolaenko, A. P. and Hayakawa, M.: Resonances in the EarthIonosphere Cavity, Kluwer Acad. Pub., Dordrecht, pp. 380, 2002.

Nickolaenko, A. P., Hayakawa, M., Sekiguchi, M., Ando, Y., and Ohta, K.: Model modifications in Schumann resonance intensity caused by a localized ionosphere disturbance over the earthquake epicenter, Ann. Geophys., 24, 567-575, 2006, http://www.ann-geophys.net/24/567/2006/.

Nickolaenko, A. P. and Hayakawa, M.: Recent studies of Schumann resonance and ELF transients, J. Atmos. Electr., 27, 19-39, 2007.

Ohta, K., Watanabe, N., and Hayakawa, M.: Survey of anomalous Schumann resonance phenomena observed in Japan, in possible association with earthquakes in Taiwan, Phys. Chem. Earth, 31, 397-402, 2006.

Ohta, K., Umeda, K., Watanabe, N., and Hayakawa, M.: ULF/ELF emissions observed in Japan, possibly associated with the ChiChi earthquake in Taiwan, Nat. Hazards Earth Syst. Sci., 1, 3742, 2001, http://www.nat-hazards-earth-syst-sci.net/1/37/2001/.

Schekotov, A. Y., Molchanov, O. A., Hayakawa, M., Fedorov, E. N., Chebrov, V. N., Sinitsin, V. I., Gordeev, E. E., Belyaev, G. G., and Yagova, N. V.: ULF/ELF magnetic field variations from atmosphere induced by seismicity, Radio Sci., 42, RS6S90, doi:10.1029/2005RS003441, 2007.

Sekiguchi, M., Hobara, Y., and Hayakawa, M.: Diurnal and seasonal variations in the Schumann resonance parameters at Moshiri, Japan, J. Atmos. Electr., 28, 1-10, 2008.

Williams, E. R., Mushtak, V., and Nickolaenko, A. P.: Distinguishing ionospheric models using Schumann resonance spectra, J. Geophys. Res., 111, D16107, doi:10.1029/2005JD006944, 2006. 